\title{
Cosmological Measurements of Dark Energy and Dark Matter
}

\author{
Segev BenZvi* \\ Department of Physics and Astronomy, University of Rochester, Rochester, NY, USA \\ E-mail: sbenzvieur.rochester.edu
}

Since the 1990s, a wealth of astrophysical measurements have demonstrated that we live in a universe dominated by dark energy and dark matter. The data, which range over several orders of magnitude in length scale and originate from across the observable universe, are well-fit by a model parametrization called $\Lambda \mathrm{CDM}$, which describes a universe of cold dark matter and a cosmological constant. However, while cosmological data sets agree broadly with $\Lambda \mathrm{CDM}$, the model provides no insight about the fundamental nature of either dark energy or dark matter. During the 2020s, a raft of new astronomical surveys will produce cosmological measurements of unprecedented precision and provide sensitive new probes of dark energy and gravitation. The new data will also yield information about the microphysics of dark matter, and allow tests of cosmological models beyond $\Lambda \mathrm{CDM}$. In this contribution, we describe the cosmological measurements made possible with the next generation of large galaxy redshift surveys, and focus in particular on the upcoming Dark Energy Spectroscopic Instrument (DESI).

XXIX International Symposium on Lepton Photon Interactions at High Energies - LeptonPhoton2019 August 5-10, 2019

Toronto, Canada

${ }^{*}$ Speaker. 


\section{Introduction}

On cosmological length scales the universe can be described as homogeneous and perfect fluid in expanding space. The evolution of the fluid is governed by General Relativity according to an "equation of motion" known as the Friedmann Equation [1]:

$$
\left(\frac{\dot{a}}{a}\right)^{2}=\Omega_{m} a^{-3}+\Omega_{\Lambda} a^{-3(1+w)}+\Omega_{r} a^{-4}+\Omega_{k} a^{-2} .
$$

Here $a$ is the scale factor of the universe at a given time, and $H(a)=\dot{a} / a$ is the Hubble parameter describing the rate of expansion. The terms on the right side of eq. (1.1) describe the energy density of matter, dark energy, radiation, and curvature respectively. Today the universe appears to be dominated by the first two components: matter, with a mean density given by the sum of "cold" (nonrelativistic) dark matter and baryonic matter, $\Omega_{m}=\Omega_{c}+\Omega_{b}$; and dark energy $\Omega_{\Lambda}$, described by an equation of state parameter $w$. A constant dark energy density based on a cosmological constant $\Lambda$ assumes $w$ is fixed at -1 .

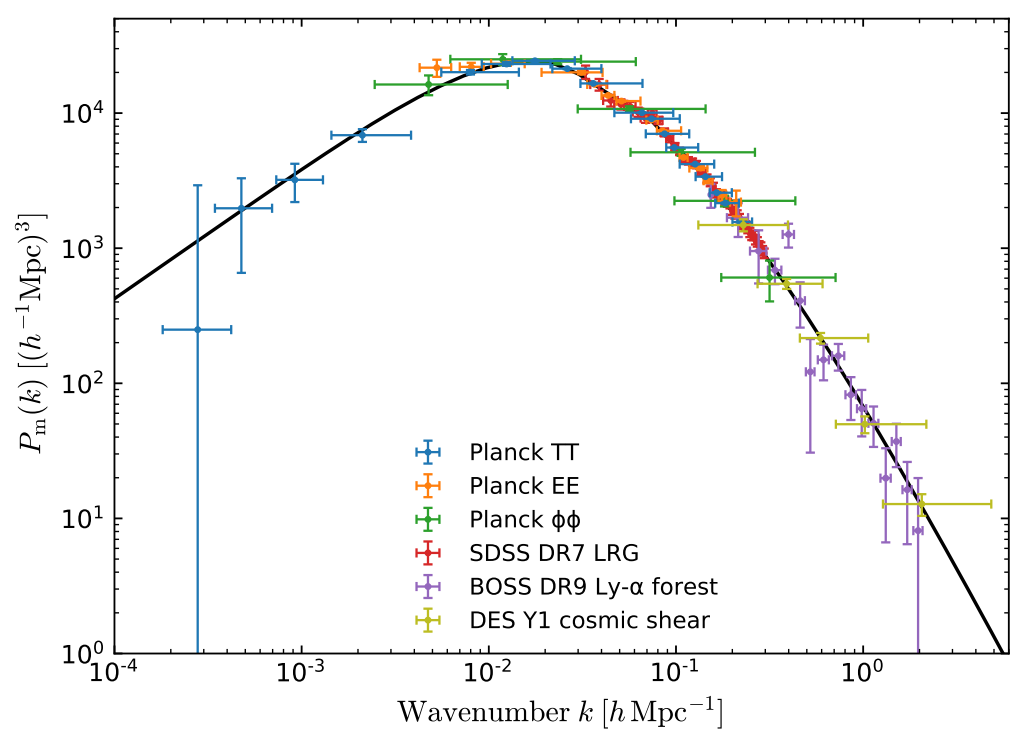

Figure 1: The linear matter power spectrum at $z=0$ using data at a variety of size scales from across the observable universe: the cosmic microwave background, galaxy redshift and Lyman$\alpha$ surveys, and weak lensing. The black line shows fit a fit using the $\Lambda \mathrm{CDM}$ model parametrization. The figure was taken from the 2018 overview of results from the Planck mission [2].

Observations sensitive to the evolution of the universe range from the cosmic microwave background (CMB), the fossil radiation from the Big Bang emitted 13.7 Gyr ago at redshift $z \approx 1100$, to galaxies and quasars observed at redshifts $z \lesssim 3$. Figure 1 summarizes the data in terms of the matter power spectrum observed today $(z=0)$ [2]. Despite covering more than 3 orders of magnitude in length scale and $14 \mathrm{Gyr}$ in time, and exhibiting very different systematic uncertainties, the data are described quite well by the 6-parameter $\Lambda \mathrm{CDM}$ model. The free parameters of $\Lambda \mathrm{CDM}$ are the baryon and dark matter densities, the age of the universe, the spectral index of primordial scalar fluctuations, the amplitude of primordial curvature fluctuations, and the optical depth at reionization (see [3] for a summary of current best-fit values). Fixed parameters in $\Lambda$ CDM include the dark energy equation of state $w=-1$, the total density $\Omega_{\text {tot }} \approx \Omega_{m}+\Omega_{\Lambda}=1$, the effective number of relativistic particle species $N_{\text {eff }}=3.046$ (assumed to be active neutrinos and derived using the Standard Model) and the sum of the neutrino masses $\sum m_{v}=0.06 \mathrm{eV}$. 


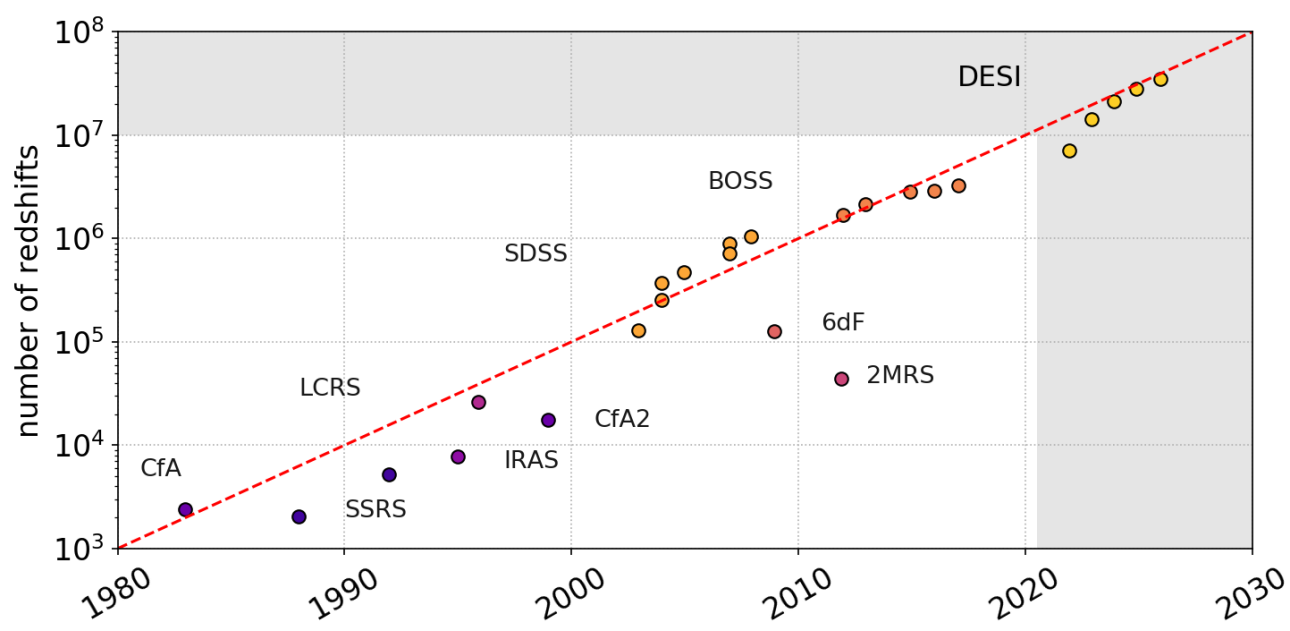

Figure 2: Measurement statistics from selected large galaxy redshift surveys, adapted from [4].

Observations of large-scale structure (LSS) with galaxies and galaxy clusters probe dark matter, gravitation, and dark energy in several ways. First, they can be used to trace the expansion of the universe via measurements of standard candles (e.g., Type Ia supernovae), standard sirens (i.e., gravitational waves from compact binary coalescence), and standard rulers such as baryon acoustic oscillations (BAO). Second, anisotropies in the BAO, known as redshift space distortions (RSDs) and caused by the peculiar motion of galaxies near massive clusters, trace the growth of structure. Present measurements of large scale structure based on $\sim 10^{6}$ galaxies and quasars are consistent with $\Lambda \mathrm{CDM}$. However, the constraints are still statistics-limited, and in the next half-decade they will shrink dramatically as the number of measured galaxy redshifts increases to $10^{7}$ (see Fig. 2). In Section 2 we discuss the new measurements and their sensitivities in the context of DESI.

\section{Measuring Dark Energy and the Growth of Structure with DESI}

The DESI instrument, currently entering its commissioning phase on the 4-m Mayall Telescope at Kitt Peak National Observatory, is a galaxy redshift survey comprising 5,000 robotically positioned optical fibers viewing a field of $8 \mathrm{deg}^{2}$. The fibers transmit light from the focal plane of the telescope to ten 3-filter spectrographs sensitive to wavelengths between $360 \mathrm{~nm}$ and $980 \mathrm{~nm}$, and with an average spectral resolution of $\lambda / \Delta \lambda \approx 3,000$ [19]. Between mid-2020 and mid-2025, DESI will survey $14,000 \mathrm{deg}^{2}$ up to a redshift $z=3.5$ using four tracers of large-scale structure:

1. 10 million spectra from galaxies brighter than $r \simeq 19.5$ between $0.05<z<0.4$, producing a magnitude-limited Bright Galaxy Survey (BGS).

2. 4 million luminous red galaxies (LRGs) between $0.4<z<1.0$, with redshift identification using the $4,000 \AA$ A break.

3. 17.1 million emission-line galaxies (ELGs) between $0.6<z<1.7$, with redshift identification using H-II emission and the 3726-3729 $\AA$ OII doublet.

4. 1.7 million quasars (QSOs) from $0.9<z<2.1$, plus 0.7 million Lyman- $\alpha$ quasars between $2.1<z<3.5$ to measure the Ly- $\alpha$ forest. 

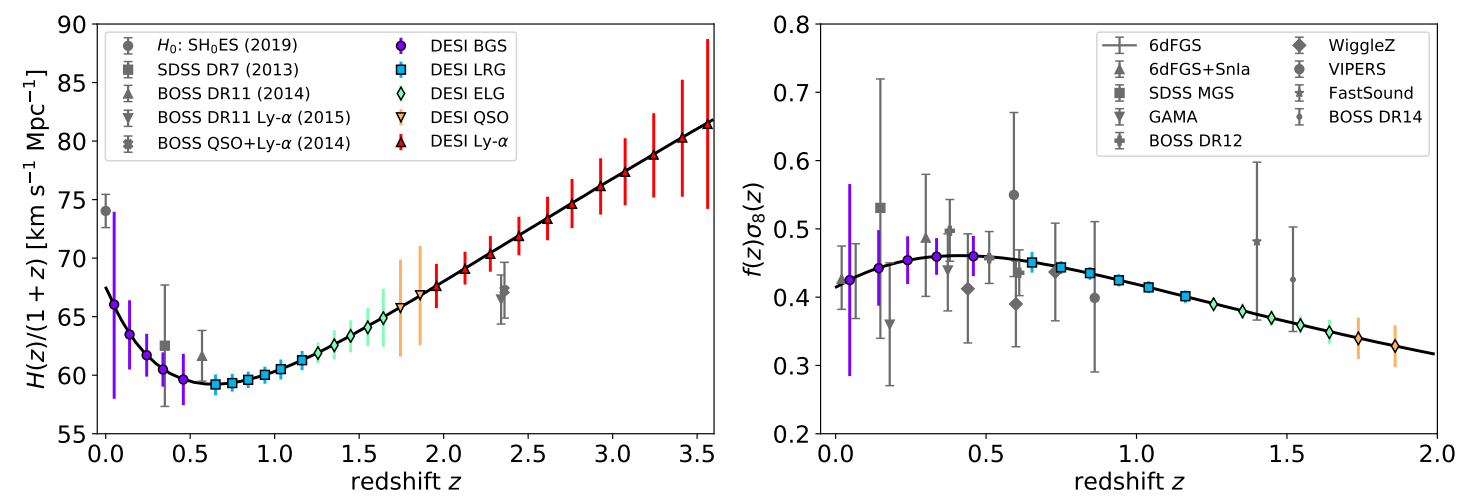

Figure 3: Left: Measurements of the Hubble expansion as a function of redshift [5, 6, 7, 8, 9], along with the projected sensitivity of DESI galaxy catalogs [10] assuming the $\Lambda$ CDM model (black line). Right: Measurements of the growth of structure as a function of redshift $[11,12,13,14,15,16,17$, 18], as well as the sensitivity of DESI catalogs [10] assuming $\Lambda$ CDM (black line).

The redshift range and projected sensitivity of DESI are illustrated in Fig. 3. The left panel shows the Hubble parameter $H$ as a function of $z$ assuming $\Lambda$ CDM (solid black line). Four recent measurements of $H$ at well-separated redshifts are indicated by the gray points. The measurement of $H$ today, $H_{0}$, at $z \approx 0$ is based on the calibration of Type Ia standard candles using the astronomical distance ladder [9], and exhibits a $\sim 4 \sigma$ discrepancy between $\Lambda$ CDM and "local" cosmological measurements. We will return to this discrepancy in Section 4. The remaining measurements come from surveys of large-scale structure, and are in reasonable agreement with $\Lambda \mathrm{CDM}$. The colored points indicate the detailed redshift coverage and the statistical power of the four tracer catalogs measured by DESI.

The right panel of Fig. 3 shows measurements of the growth of structure, parametrized by the growth rate $f(z)$ and the (normalized) amplitude of mass fluctuations $\sigma_{8}(z)$. Again, the black line indicates the prediction of $\Lambda \mathrm{CDM}$, and is consistent with measurements of large-scale structure across a large range of redshifts, indicated by the gray points. Projected DESI measurements are shown by colored markers.

The main goals of DESI are to tightly constrain the dark energy equation of state and investigate alternative models of dark energy and gravitation. Although current data are consistent with a cosmological constant, an open question is whether or not dark energy evolves with time - for example [20],

$$
w(z)=w_{0}+w_{a}(1-a),
$$

where $w_{0}$ is the dark energy equation of state today and $w_{a}$ describes its time evolution with redshift. The full DESI survey is projected to achieve a factor of 20 improvement in constraints on the dark energy equation of state with respect to previous surveys ${ }^{1}$ [10]. A second open question is whether or not General Relativity fully describes gravitation at cosmological length scales, or if the data favor modified theories of gravity. For example, the growth rate of structure can be parametrized

\footnotetext{
${ }^{1}$ As measured using the Dark Energy Task Force figure of merit $\left[\operatorname{det}\left\{\operatorname{cov}\left(w_{0}, w_{a}\right)\right\}\right]^{-1 / 2}$.
} 
in terms of $\Omega_{m}$ and a growth index $\gamma$,

$$
f(z) \approx \Omega_{m}(z)^{\gamma},
$$

with General Relativity (GR) consistent with $\gamma=0.55+0.05[1+w(z=1)]$ [21]. Measurements of $\gamma$ that differ from 0.55 would provide important evidence that GR is not complete at cosmological distances, and DESI should be able to rule out GR at the $3 \sigma$ level after two years of data taking $[10,22]$. DESI achieves this sensitivity using complementary measurements of BAO and RSDs, described in Section 2.1.

\subsection{Baryon Acoustic Oscillations and Redshift Space Distortions}

Baryon acoustic oscillations, or BAO, are features in the statistical distribution of galaxies on large scales. They correspond to the maximum distance travelled by acoustic waves in the photonmatter plasma prior to decoupling at $z \approx 1100$. At this distance a small overdensity at the sound horizon (the rims of photon-matter "bubbles" in the plasma) evolved into a small overdensity in the distribution of galaxies. Today we measure $\mathrm{BAO}$ as a peak in the 3D separation of galaxies at a comoving distance of $s=110 \mathrm{~h}^{-1} \mathrm{Mpc}$. The peak, visible in the two-point correlation function of tracer galaxies and as a series of "wiggles" in the matter power spectrum, is illustrated in the left panel of Fig. 4. The BAO peak appears as a ring-like structure since the correlation function is plotted as a function of distance parallel and perpendicular to the line of sight.
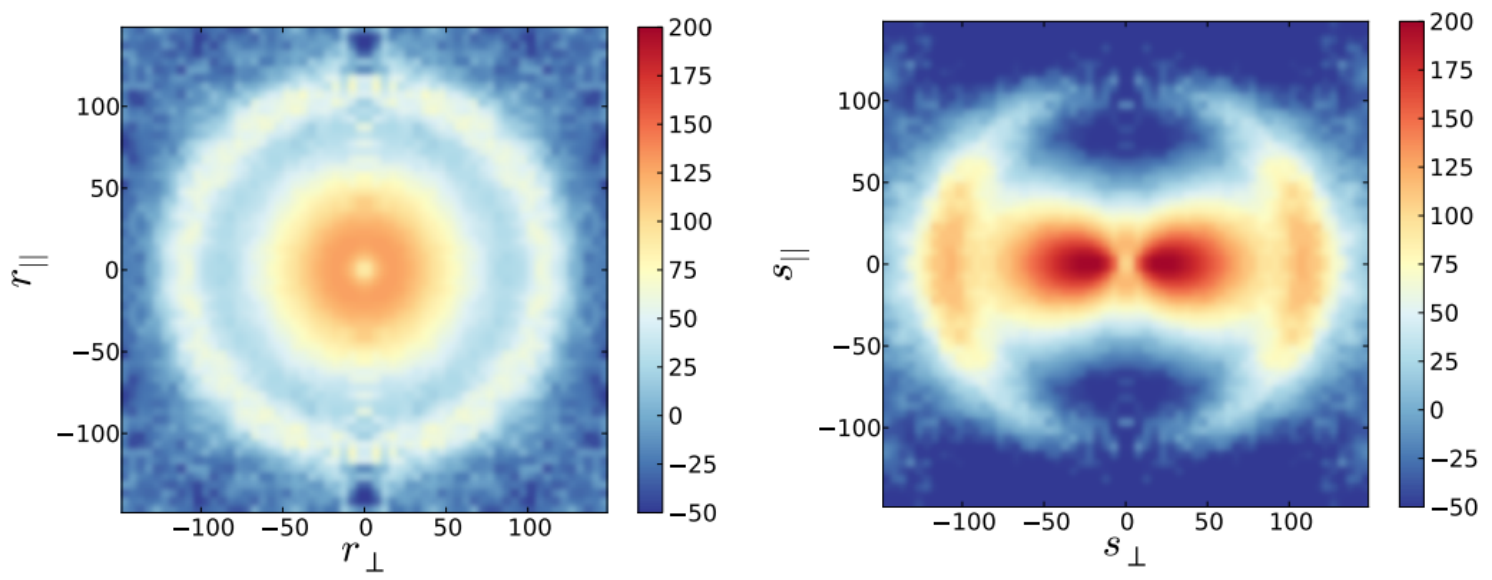

Figure 4: Simulated redshift space distortions (RSD) using 160 mock galaxy catalogs (figure from [23]). Left: the two-point galaxy correlation $r^{2} \xi(r)$ in real space as a function of separation along $\left(r_{\|}\right)$and perpendicular to $\left(r_{\perp}\right)$ the line of sight. Right: the two-point correlation $s^{2} \xi(s)$ in redshift space as a function of comoving separation. The RSDs manifest as the anisotropy (the ellipticity or "squashing") of the correlation function along the line of sight $s \|$.

The sound horizon is well-known and thus the BAO feature is usable as a standard ruler to convert redshift to comoving distance. By measuring the BAO scale at a range of redshifts, it is possible to characterize the smooth expansion history of the universe $H(z)$ - the left panel of Fig. 3 - and angular diameter distance. These values provide a direct constraint on dark energy.

However, before the BAO feature can be used for cosmology, observations must be corrected for redshift space distortions (RSDs). Tracer galaxies are gravitationally attracted to clusters, so 
they exhibit peculiar velocities - significant motion on top of the flow due to the Hubble expansion - as they fall into gravitational wells. In redshift space, peculiar motion causes clusters to appear squashed along the line of sight, creating an artificial anisotropy or ellipticity in the BAO feature (see the right panel of Fig. 4). The anisotropy can be significantly mitigated using a "reconstruction" procedure in which the galaxies are used to estimate the underlying gravitational gradients producing the peculiar motion. The galaxy positions are then backtraced to the positions they would occupy if they were not falling into clusters, thus recovering the isotropy of the BAO. For a detailed summary of the reconstruction procedure and its applicability at cosmological distance scales above the "nonlinear" regime, see [23] and references therein.

Although RSDs contaminate measurements of the Hubble expansion using BAO, they also provide crucial information about peculiar velocities and the growth of structure. Thus, measuring RSDs with different galaxy catalogs probes $f \sigma_{8}$ vs. $z$. The projected sensitivity of DESI to the dark energy equation of state with BAO and the growth of structure with RSDs is shown in Fig. 5.
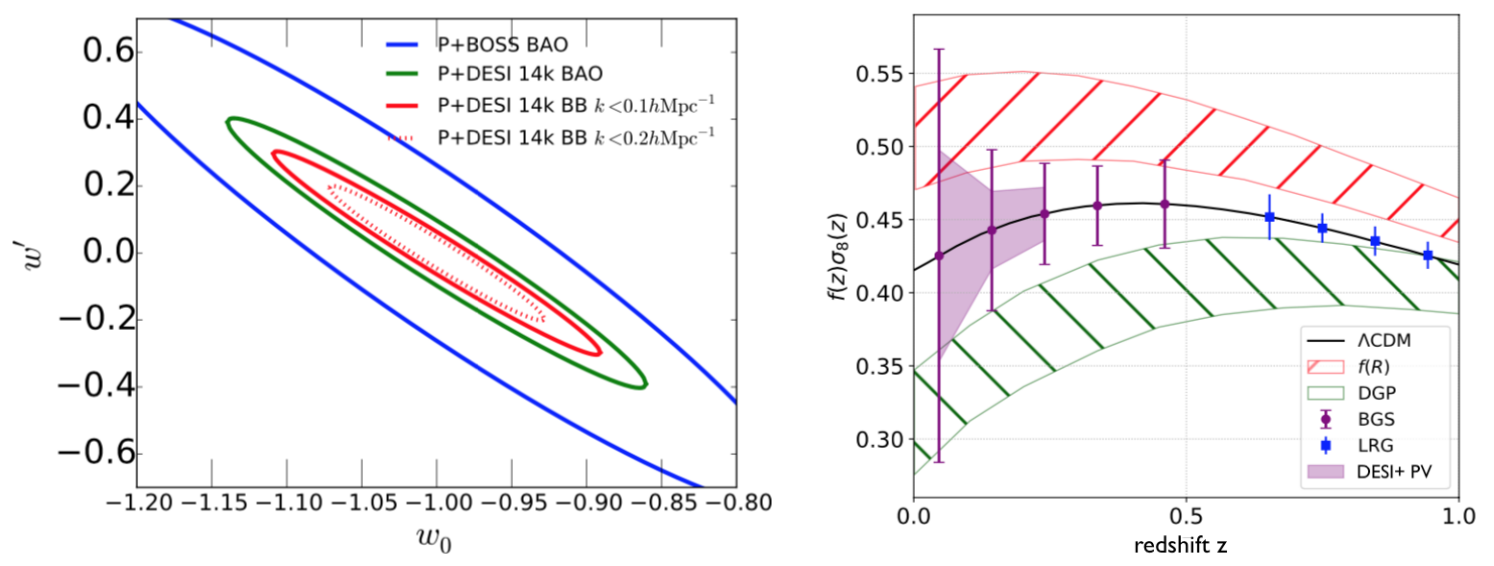

Figure 5: Left: joint dark energy constraints on $w_{0}$ and $w^{\prime}\left(w_{a}\right)$ with existing data from BOSS (blue contour) and improvements expected with DESI (green and red contours) [10]. Right: sensitivity of DESI to $f \sigma_{8}$ using RSDs from the BGS and LRG catalogs, as well as a peculiar velocity survey using standard candles (purple filled area). The solid black line indicates $\Lambda \mathrm{CDM}$; the hatched regions show the predicted $f \sigma_{8}$ from two alternative models to GR. Adapted from [10, 22, 24].

\section{The Growth of Structure and Dark Radiation}

Although $\Lambda \mathrm{CDM}$ is not sensitive to the microphysics of dark matter, the growth of structure particularly non-linear growth at small length scales $\left(k \gtrsim 0.5 h \mathrm{Mpc}^{-1}\right)$ - can give important clues about the particle nature of dark matter. This perhaps best illustrated by considering primordial neutrinos and their effect on structure above the peak in the matter power spectrum.

A significant background of relic neutrinos from the Big Bang (the cosmic neutrino background, or $\mathrm{C} v \mathrm{~B}$ ) makes up the overwhelming majority of neutrinos in the universe. Due to their extremely low mass, the $\mathrm{C} v \mathrm{~B}$ particles acted like relativistic ("hot") dark matter in the early universe, escaping the potential wells formed by primordial density fluctuations. At later times, the neutrinos cooled and contributed to $\Omega_{m}$ [25]. Neutrino free streaming thus suppresses the growth 
of density fluctuations as the universe evolves. Moreover, the effect of relativistic particles on the evolution of structure constrains their fundamental properties.
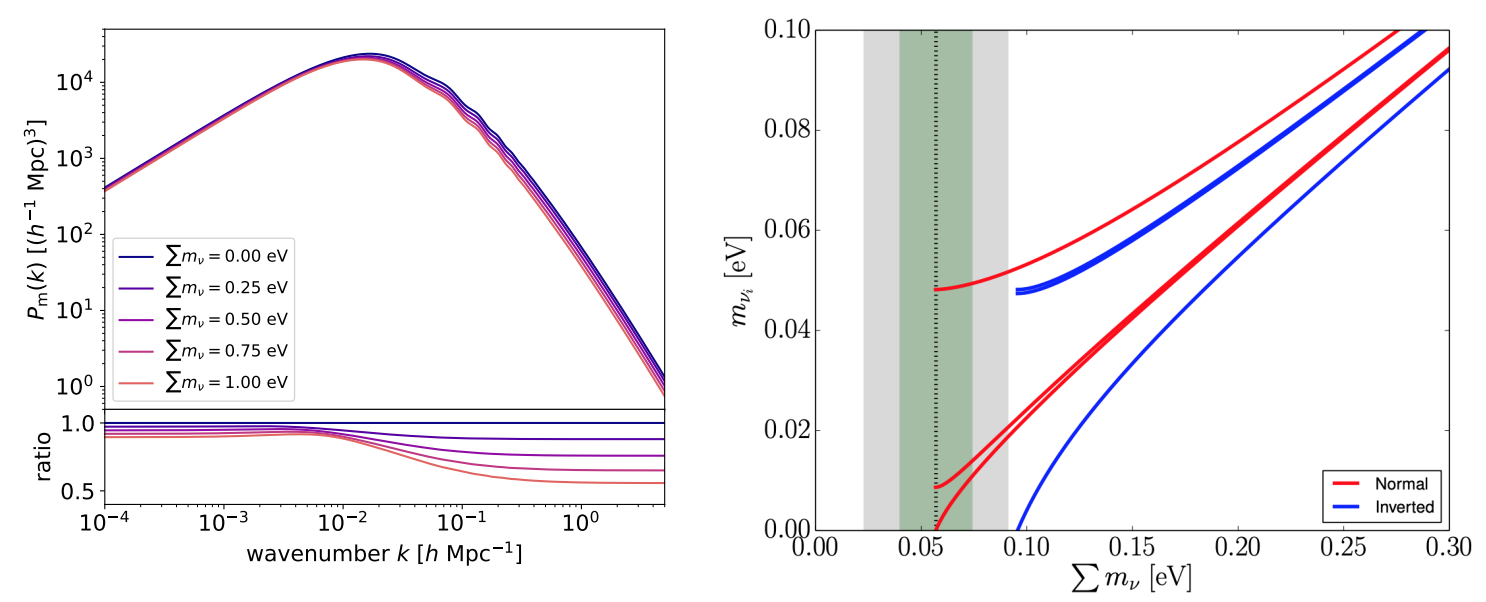

Figure 6: Left: sensitivity of the matter power spectrum to $\sum m_{v}$; adapted from [25]. Right: projected sensitivity of DESI to the sum of the neutrino masses assuming normal and inverted mass ordering using the Ly- $\alpha$ forest [26].

Figure 6 shows the impact of the sum of the neutrino masses $\sum m_{v}$ the matter power spectrum $P_{m}(k)$, with the bottom of the left panel indicating the ratio $P_{m}(k) / P_{m}\left(k, \sum m_{v}=0\right)$. At early times the free-streaming $\mathrm{C} v \mathrm{~B}$ reduces the formation of small-scale structure, but later becomes nonrelativistic and acts like cold dark matter. The transition occurs at $z \sim 2000 m_{v} / 1 \mathrm{eV}$, near the peak in the matter power spectrum [25]. Larger values of $\sum m_{v}$ increase the suppression of small-scale structure since the energy density $\Omega_{v}$ becomes a larger contribution to the total matter density $\Omega_{m}$ after the relativistic to non-relativistic transition. Measurements of the matter power spectrum are thus sensitive to $\sum m_{v}$, particularly at large values of $k$ where the suppression of structure is most pronounced. The right panel of Fig. 6 shows the expected sensitivity of DESI and the next generation of CMB experiments to $\sum m_{v}$ using the Ly- $\alpha$ forest [26]. Combined CMB and structure measurements will yield a measurement resolution of $\sigma_{\sum m_{v}} \approx 17 \mathrm{meV}$. Neutrino oscillations require that $\sum m_{v}>59 \mathrm{meV}$ (normal mass ordering) or $>98 \mathrm{meV}$ (inverted ordering). Thus, if $\sum m_{v}$ is very small cosmological measurements will rule out the inverted hierarchy at $3.5 \sigma$.

Large-scale structure is also sensitive to additional particles beyond the Standard Model. For example, while $\Lambda \mathrm{CDM}$ assumes only the presence of photons and the known active neutrino species ( $N_{\text {eff }}=3.046$ ), observations of $N_{\text {eff }}>3.046$ could be evidence for sterile neutrinos or other light relic particles ("dark radiation"). Increasing $N_{\text {eff }}$ increases structure formation on small scales, effectively because it decreases the ratio $\Omega_{b} / \Omega_{c}$ (see the detailed discussion in [27]). Relative to $\Lambda \mathrm{CDM}$, this increases clumping of cold dark matter before decoupling (increasing small-scale structure growth) and suppresses the BAO feature. Allowing $N_{\text {eff }}>3$ in fits of CMB+LSS data does reduce tension in measurements of $H_{0}$ between cosmological data sets and the local distance ladder (e.g., $[28,29]$ ), while also increasing $\sigma_{8}$. However, current constraints favor the $\Lambda$ CDM values. Within the next 5 years, DESI + Stage IV CMB measurements will reduce $\sigma_{N_{\text {eff }}}$ by a factor of 9 over current data sets [25]. 


\section{Hints of Physics Beyond $\Lambda$ CDM, or Systematic Uncertainties?}

As discussed in Section 2, discrepancies have emerged during the past five years in measurements of $H_{0}$, the value of the Hubble expansion rate today, between cosmological data sets and the observations using the astronomical distance ladder. As of the time of this conference, the statistical significance of the discrepancy is $\sim 4 \sigma$, resulting in considerable speculation about the origin of the difference: unknown systematic uncertainties, or cracks in the $\Lambda$ CDM model.

The ultimate answer to this question must wait until the future. However, we note that the $H_{0}$ tension has stimulated a number of new measurement techniques with independent systematic uncertainties, ranging from additions to the traditional distance ladder [30] to multi-messenger observations of standard sirens [31,32]. In this section we discuss several measurements of $H_{0}$ that are possible using large-scale structure, and provide projected uncertainties over the lifetime of DESI.

\subsection{Constraining $H_{0}$ with BAO}
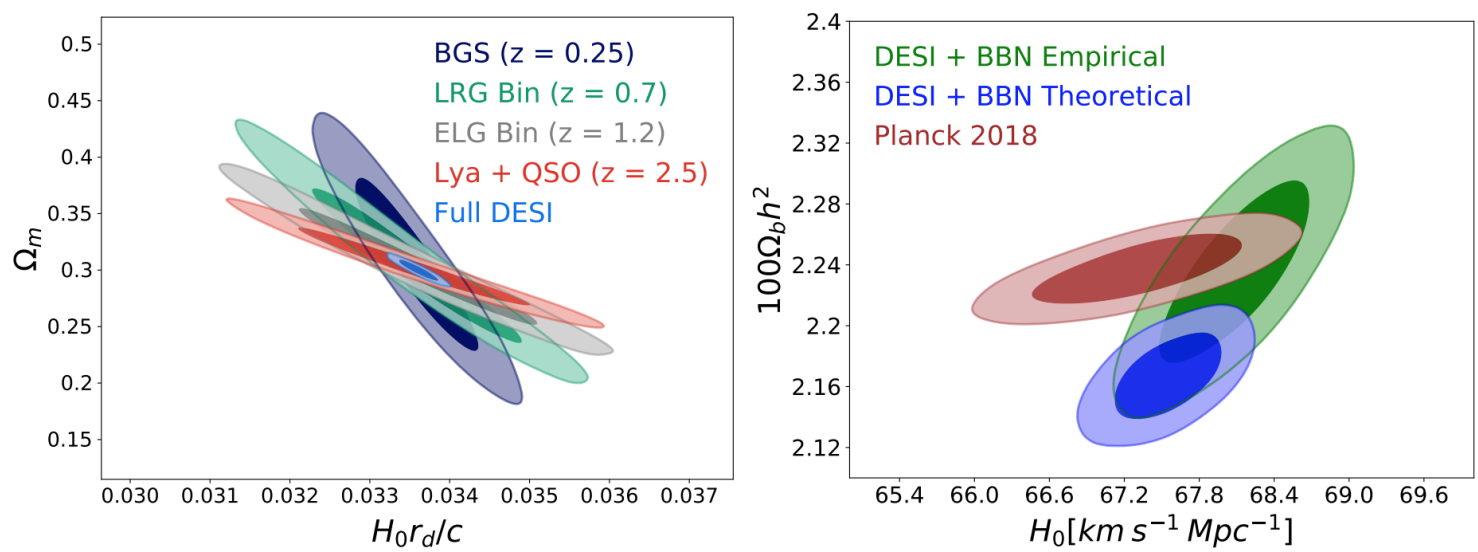

Figure 7: Left: contours in $\Omega_{m}-H_{0} r_{d}$ expected from DESI. Right: constraints on $H_{0}$ using BAO, with $r_{d}$ estimated using the abundance of primordial deuterium. Figure from [33].

Recent estimates of $H_{0}$ with $\mathrm{BAO}$, independent of the $\mathrm{CMB}$, were presented in [33]. In brief, given eq. (1.1), the fact that $\dot{a} / a=H(z) / H_{0}$, and the constraint $\Omega_{\Lambda}=1-\Omega_{m}$, the free parameters of $H(z)$ are $H_{0}$ and $\Omega_{m}$. Measurements of BAO perpendicular to the line of sight constrain the combination $D_{M}(z) / r_{d}$, where $D_{M}(z)$ is the comoving angular diameter distance and $r_{d}$ is the size of the sound horizon at the drag epoch when baryons completely decouple from photons. The BAO peak along the line of sight measures the combination $c / H(z) r_{d}$. Thus the BAO peak constrains $H(z)$ in the $\Omega_{m}-H_{0} r_{d}$ plane. The projected 2D contours in this plane using the DESI galaxy catalogs are shown in the left panel of Fig. 7, along with a joint constraint from all four catalogs [33].

Breaking the $H_{0}-r_{d}$ degeneracy requires an estimate of $r_{d}$, which depends on the density of neutrinos, matter, and baryons. The right panel of Fig. 7 shows the constraint on $H_{0}$ if an estimate of $r_{d}$ is made using $\Omega_{m}$ from BAO, $\Omega_{b}$ from the abundance of primordial deuterium (BBN), and $\Omega_{v}$ is fixed using the $\Lambda$ CDM values of $\sum m_{v}$ and $N_{\text {eff }}$ [33]. This work suggests that competitive constraints on $H_{0}$ are possible using $\mathrm{BAO}$, independent of the $\mathrm{CMB}$. A preliminary measurement of $H_{0}$ using data from BOSS is in $\sim 3 \sigma$ disagreement with the distance ladder [33]. 


\subsection{Constraining $H_{0}$ with Bright and Dark Sirens}

Compact binary coalescence events, which emit significant power into gravitational waves (GWs), were first suggested as a tool to measure $H_{0}$ over 30 years ago [34]. The advantage of the technique is that it is fully independent of the astronomical distance ladder. A binary's orbital frequency and frequency sweep ("chirp”) entirely determine its GW luminosity. Thus, a calibrated measure of the GW strain at Earth provides a measurement of the luminosity distance to the source, analogous to photon flux measurements performed with standard candles. Using an acoustic metaphor for the measurement, such events are called "standard sirens" if the redshift of the host galaxy is known.

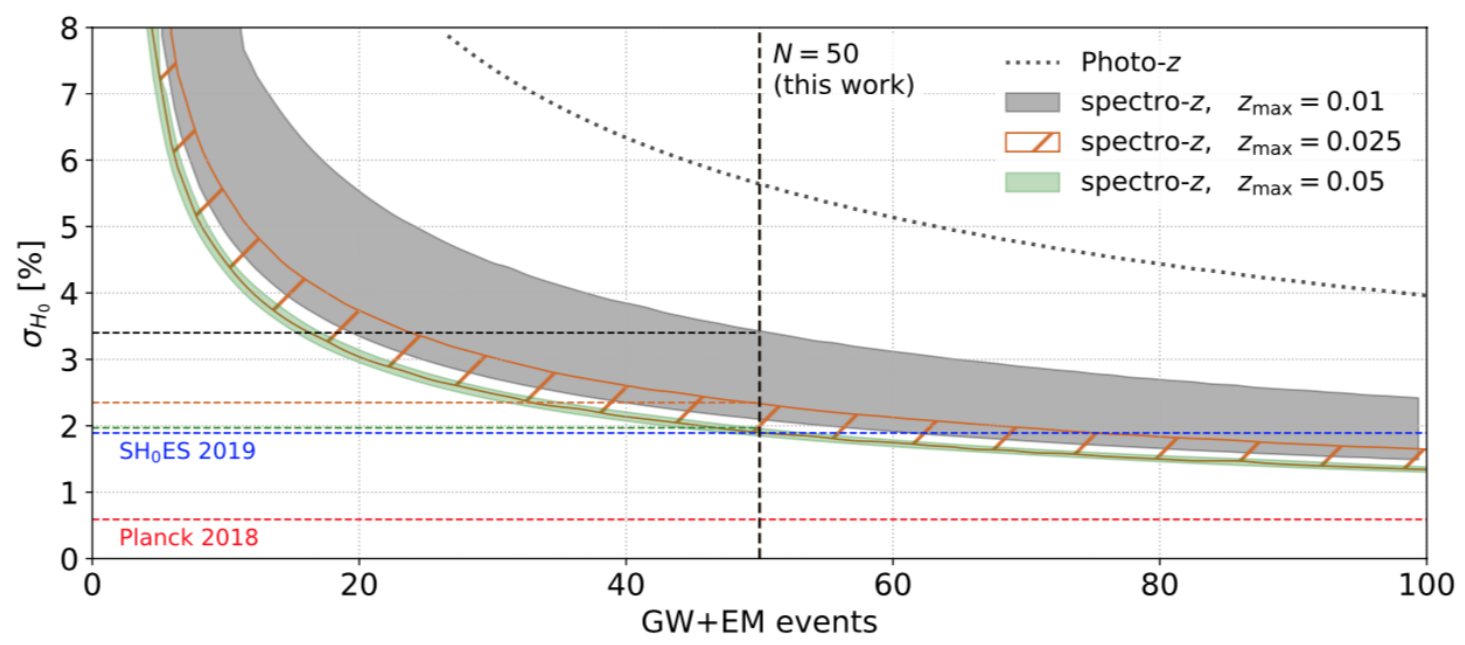

Figure 8: Relative cumulative uncertainty on $H_{0}$ after $n$ measurements of bright sirens, assuming three surveys with maximum redshift $z_{\max }$. The upper and lower edges of the filled bands show the contribution due to pessimistic and optimistic values of the hosts' peculiar velocity uncertainties. The dotted line shows the reduction of the constraints if only photometric redshifts are available. Figure adapted from [35] and [36].

Measurements of large-scale structure can provide significant improvements to constraints on $H_{0}$ from binary coalescence events. Two cases are possible:

1. An electromagnetic counterpart to the GW event, as in the case of GW170817 [31], allows identification of the host galaxy and its redshift. In the case of such a bright siren, a dominant systematic uncertainty in $H_{0}$ comes from the peculiar velocity of the host. Catalogs of redshift measurements of galaxies in the volume around the host galaxy thus considerably reduce this uncertainty [36].

2. No electromagnetic counterpart, and therefore no host galaxy, is observed. For such a dark siren, $H_{0}$ can be estimated by integrating over a large number of potential host galaxies in the GW localization volume [32]. A dominant systematic uncertainty in $H_{0}$ is the precision in the redshifts of host candidates $[32,36]$.

Figure 8 shows the cumulative relative uncertainty in $H_{0}$ from bright siren events with redshifts measured photometrically and spectroscopically, for three GW catalogs with a maximum 
redshift $z_{\max }$. The edges of the filled bands show the effects of peculiar velocity uncertainties in the host redshift, with the upper edge of each band assuming $\sigma_{\mathrm{PV}}=400 \mathrm{~km} \mathrm{~s}^{-1}$ and the lower band assuming $100 \mathrm{~km} \mathrm{~s}^{-1}$. The relative improvement in $\sigma_{H_{0}}$ when spectroscopic redshifts are available applies equally well to the case of dark sirens.

During the next five years, the DESI BGS catalog will provide a volume-limited sample of galaxies over $14,000 \mathrm{deg}^{2}$ up to $z \simeq 0.2$, and a magnitude-limited sample up to $z=0.4$. The catalog should prove extremely useful for mitigating measurement uncertainties in $H_{0}$ from both bright and dark sirens. For bright sirens, the BGS will constrain the peculiar velocities of nearly all likely GW hosts in the nearby universe. For dark sirens, the DESI BGS will substantially reduce the redshift uncertainties of host candidates. Constraints on $H_{0}$ after the five-year DESI survey from both types of events are projected at the $2 \%$ level [36].

\section{Conclusion}

All current evidence for dark matter and dark energy comes from astrophysical data sets. Dark energy is measured using the expansion of the universe and the growth of large-scale structure, while dark matter is probed by structure at small size scales. Present cosmological measurements are in broad agreement with the $\Lambda \mathrm{CDM}$ model, but the inauguration of large spectroscopic and photometric surveys, as well as the start of experiments such as CMB-S4, will improve constraints on model parameters by a factor of 10 or more during the next decade. The data will be extremely useful for detailed studies of systematic differences between cosmological measurements and observations of the local universe. More importantly, they will enable studies of the nature of dark energy and gravitation that were heretofore impossible. Is dark energy really described by a cosmological constant? Is GR a sufficient theory of gravitation on the largest length scales? We hope to be much closer to answering these questions by 2025 . Stay tuned!

\section{Acknowledgments}

The author is grateful to the conference organizers for the invitation to Toronto, and thanks them for constructing a very engaging scientific program. This work has been supported by the U.S. Department of Energy Office of High Energy Physics under award number DE-SC0008475.

\section{References}

[1] R. J. Nemiroff and B. Patla, Am. J. Phys. 76 (2008) 265-276.

[2] Planck Collaboration, Y. Akrami et al., arXiv:1807.06205.

[3] Planck Collaboration, N. Aghanim et al., arXiv:1807.06209.

[4] D. J. Schlegel et al., arXiv:1907.11171.

[5] X. Xu, A. J. Cuesta, N. Padmanabhan, D. J. Eisenstein, and C. K. McBride, Mon. Not. Roy. Astron. Soc. 431 (2013) 2834.

[6] BOSS Collaboration, L. Anderson et al., Mon. Not. Roy. Astron. Soc. 441 (2014) 24-62.

[7] BOSS Collaboration, A. Font-Ribera et al., JCAP 1405 (2014) 027. 
[8] BOSS Collaboration, T. Delubac et al., Astron. Astrophys. 574 (2015) A59.

[9] A. G. Riess, S. Casertano, W. Yuan, L. M. Macri, and D. Scolnic, Astrophys. J. 876 (2019) 85.

[10] DESI Collaboration, arXiv:1611.00036.

[11] F. Beutler, C. Blake, M. Colless, D. H. Jones, L. Staveley-Smith, L. Campbell, Q. Parker, W. Saunders, and F. Watson, Mon. Not. Roy. Astron. Soc. 416 (2011) 3017-3032.

[12] C. Blake et al., Mon. Not. Roy. Astron. Soc. 425 (2012) 405-414.

[13] C. Blake et al., Mon. Not. Roy. Astron. Soc. 436 (2013) 3089.

[14] C. Howlett, A. Ross, L. Samushia, W. Percival, and M. Manera, Mon. Not. Roy. Astron. Soc. 449 (2015) 848-866.

[15] T. Okumura et al., Publ. Astron. Soc. Jap. 68 (2016) 38.

[16] A. Pezzotta et al., Astron. Astrophys. 604 (2017) A33.

[17] D. Huterer, D. Shafer, D. Scolnic, and F. Schmidt, JCAP 1705 (2017) 015.

[18] P. Zarrouk et al., Mon. Not. Roy. Astron. Soc. 477 (2018) 1639-1663.

[19] DESI Collaboration, arXiv:1611.00037.

[20] M. Chevallier and D. Polarski, Int. J. Mod. Phys. D10 (2001) 213-224.

[21] E. V. Linder, Phys. Rev. D72 (2005) 043529.

[22] A. G. Kim et al., arXiv:1903.07652.

[23] N. Padmanabhan, X. Xu, D. J. Eisenstein, R. Scalzo, A. J. Cuesta, K. T. Mehta, and E. Kazin, Mon. Not. Roy. Astron. Soc. 427 (2012) 2132-2145.

[24] L. Samushia et al., Mon. Not. Roy. Astron. Soc. 429 (2013) 1514-1528.

[25] K. N. Abazajian et al., Astropart. Phys. 63 (2015) 66-80.

[26] A. Font-Ribera, P. McDonald, N. Mostek, B. A. Reid, H.-J. Seo, and A. Slosar, JCAP 1405 (2014) 023.

[27] Particle Data Group Collaboration, M. Tanabashi et al., Phys. Rev. D98 (2018) 030001.

[28] Planck Collaboration, P. A. R. Ade et al., Astron. Astrophys. 594 (2016) A13.

[29] C. D. Kreisch, F.-Y. Cyr-Racine, and O. Doré, arXiv:1902.00534.

[30] W. L. Freedman et al., arXiv:1907.05922.

[31] LIGO Scientific, Virgo, 1M2H, Dark Energy Camera GW-E, DES, DLT40, Las Cumbres Observatory, VINROUGE, MASTER Collaboration, B. P. Abbott et al., Nature 551 (2017) 85-88.

[32] DES, LIGO Scientific, Virgo Collaboration, M. Soares-Santos et al., Astrophys. J. 876 (2019) L7.

[33] A. Cuceu, J. Farr, P. Lemos, and A. Font-Ribera, arXiv:1906.11628.

[34] B. F. Schutz, Nature 323 (1986) 310-311.

[35] D. J. Mortlock, S. M. Feeney, H. V. Peiris, A. R. Williamson, and S. M. Nissanke, Phys. Rev. D, in press (2019).

[36] A. Palmese et al., arXiv:1903.04730. 